Algebraic $\& \mathcal{G}$ Geometric Topology

Volume 1 (2001) 231-241

Published: 16 April 2001

ATG

\title{
Infinite Order Amphicheiral Knots
}

\author{
Charles Livingston
}

\begin{abstract}
In answer to a question of Long, Flapan constructed an example of a prime strongly positive amphicheiral knot that is not slice. Long had proved that all such knots are algebraically slice. Here we show that the concordance group of algebraically slice knots contains an infinitely generated free subgroup that is generated by prime strongly positive amphicheiral knots. A simple corollary of this result is the existence of positive amphicheiral knots that are not of order two in concordance.
\end{abstract}

AMS Classification 57M25; 57M27

Keywords Knot, amphicheiral, concordance, infinite order

\section{Introduction}

In 1977 Gordon [G] asked whether every class of order two in the classical knot concordance group can be represented by an amphicheiral knot. The question remains open although counterexamples in higher dimensions are now known to exist $[\mathrm{CM}]$. This problem is more naturally stated in terms of negative amphicheiral knots, since such knots represent 2-torsion in concordance; that is, if $K$ is negative amphicheiral, $K \# K$ is slice. On the other hand there is no reason to assume that positive amphicheiral knots represent 2-torsion. Surprisingly, until now no examples of positive amphicheiral knots that are not 2 -torsion in concordance have been known. The first goal of this paper is to present an example of a positive amphicheiral knot that is of infinite order in the concordance group.

Long [Lo] proved that every strongly positive amphicheiral knot is algebraically slice. (This result followed work of Hartley and Kawauchi [HK] showing a close relationship between strongly positive amphicheiral knots and slice knots.) Long also used an example of the author [L1] to show that such knots need not be slice. Long's example was composite and he asked whether such an example exists among prime strongly amphicheiral knots. Flapan provided such an example in $[\mathrm{F}]$. Here we will prove that examples such as Flapan's 
are of infinite order in the concordance group. This, of course, provides an example of a positive amphicheiral knot that is not 2-torsion in concordance. We will in fact prove much more, describing an infinite set of strongly positive amphicheiral knots that are linearly independent in the concordance group. We restate this as the main theorem of this paper.

Theorem 1.1 The group of concordance classes of algebraically slice knots contains an infinitely generated free subgroup generated by prime strongly positive amphicheiral knots.

Acknowledgement This research was motivated by a question of Alexander Stoimenow asking the order of Flapan's knot in the concordance group.

\section{Amphicheirality}

We will assume the reader is familiar with basic knot theory; [Ro] serves as a good reference. Material concerning Casson-Gordon invariants will be referenced as needed. Since the details of orientation and amphicheirality may be less familiar, we review them briefly here.

For our purposes it is simplest to view a knot as a smooth oriented pair, $(S, K)$, where $S$ is diffeomorphic to $S^{3}$ and $K$ is diffeomorphic to $S^{1}$. Such a pair is usually abbreviated simply as $K$. Associated to $K$ we have three other knots: the mirror of $K, K^{*}=(-S, K)$; the reverse of $K, K^{r}=(S,-K)$; and the (concordance) inverse of $K,-K=(-S,-K)$.

A knot is called positive amphicheiral if $(S, K)$ is oriented diffeomorphic to $(-S, K)$, and is called strongly positive amphicheiral if that diffeomorphism can be taken to be an involution. In terms of a knot diagram a knot is strongly positive amphicheiral if changing all the crossings of some oriented diagram of the knot results in a new diagram that can be transformed back into the original diagram by a 180 degree rotation. In Figure 2 we illustrate a strongly positive amphicheiral knot.

\section{Casson-Gordon invariant results}

To prove that algebraically slice knots are not slice we need Casson-Gordon invariants. We now summarize the results that we will use. The main result of [CG1] is the following. Let $K$ be a knot in $S^{3}$ and let $M_{q}$ denote its $q$-fold cyclic branched cover with $q$ a prime power. 
Proposition 3.1 If a knot $K$ is slice then there is a subgroup $H \subset H_{1}\left(M_{q}, \mathbf{Z}\right)$ such that:

(1) $H^{\perp}=H$ with respect to the linking form on $H_{1}\left(M_{q}, \mathbf{Z}\right)$ and, in particular, the order of $H$ is the square root of the order of $H_{1}\left(M_{q}, \mathbf{Z}\right)$.

(2) If $\chi: H_{1}\left(M_{q}, \mathbf{Z}\right) \rightarrow \mathbf{Z}_{p^{k}}, p$ prime, is homomorphism that vanishes on $H$ then the associated Casson-Gordon invariant satisfies $\sigma(K, \chi)=0$.

(3) $H$ is invariant under the group of deck transformations.

The invariance of $H$ is not stated in [CG1] but follows readily from the construction of $H$. Note that in [CG1] the invariant $\sigma(K, \chi)$ is denoted $\sigma_{1}(\tau(K, \chi))$. To use this result we do not need the explicit definition of the Casson-Gordon invariants, but need only their properties given in Propositions 3.2 and 3.3 below.

We will be constructing our examples by starting with a knot $K$ and replacing the neighborhood of an unknotted circle $L$ in the complement of a Seifert surface for $K$ with the complement of a knot $J$. The identification map switches longitude and meridian so that the resulting manifold is still $S^{3}$. The effect of this construction is to tie that portion of $K$ that passes through $L$ into a knot. Details can be found, for example, in [GL1]. Note that the construction does not change the Seifert form of $K$. Furthermore, there is a natural isomorphism between the homology groups of the $q$-fold cyclic branched covers for any given $q$. To see this a simple Mayer-Vietoris argument can be used: the branched cover of the modified knot is built from the branched cover of $K$ by removing a collection of homology circles (solid tori) and replacing them with other homology circles (copies of the complement of $J$ ). Similarly, there is a correspondence between characters on the two homology groups.

We denote the new knot by $K_{J}$. In the construction used below, this process is iterated; we start with a link $L$ and replace each component with a knot complement.

In the next proposition $\sigma_{j / p^{k}}(J)$ denotes the classical signature of $J$ given as the signature of $(1-\omega) V+(1-\bar{\omega}) V^{t}$, where $V$ is a Seifert matrix for $J$ and $\omega=e^{j 2 \pi i / p^{k}}$.

Proposition 3.2 Suppose that $K_{J}$ is obtained from $K$ by removing the neighborhood of an unknotted circle $L$ and replacing it with the complement of a knot $J$ as described above. Then $\sigma\left(K_{J}, \chi\right)-\sigma(K, \chi)=\sum_{j=0}^{q-1} \sigma_{\chi\left(T^{j}(\tilde{L})\right) / p^{k}}(J)$, where the $\tilde{L}$ is a lift of $L, T$ denotes the generator of the group of deck transformations, and $\chi: H_{1}\left(M_{q}, \mathbf{Z}\right) \rightarrow \mathbf{Z}_{p^{k}}$ is a homomorphism. 
Proof The proof is contained in [GL1]. It is based on a similar result for computing Casson-Gordon invariants of satellite knots first found by Litherland in $[\mathrm{Lt}]$

The following result is an immediate consequence of a result of Litherland in [Lt].

Proposition 3.3 If $\chi$ is the trivial character, then $\sigma(K, \chi)=0$.

Proof In Corollary B2 of [Lt] it is shown that for the trivial character $\chi$ one has $\sigma_{\xi}(K, \chi)=\sum_{\zeta^{\nu}=\xi} \sigma_{\zeta}(K)-\sum_{\omega^{\nu}=1} \sigma_{\omega}(K)$. Here $\sigma_{\xi}(K, \chi)$ is a more general Casson-Gordon invariant than what we use here; it is related to $\sigma(K, \chi)$ and the proposition follows from the formula $\lim _{\xi \rightarrow 1} \sigma_{\xi}(K, \chi)=\sigma(K, \chi)$.

\section{$4 \quad$ Basic building blocks}

Consider the knot $K_{2 m+1}$ illustrated in Figure 1. In the figure a Seifert surface $F$ for $K_{2 m+1}$ is evident. The bands are twisted in such a way that the Seifert form is

$$
V_{m}=\left(\begin{array}{cc}
0 & m+1 \\
m & 0
\end{array}\right)
$$

Notice that $K_{\mu}$ is reversible and that $-K_{\mu}=K_{-\mu}$, which has Seifert form $V_{-m-1}$ since $-2 m-1=2(-m-1)+1$.

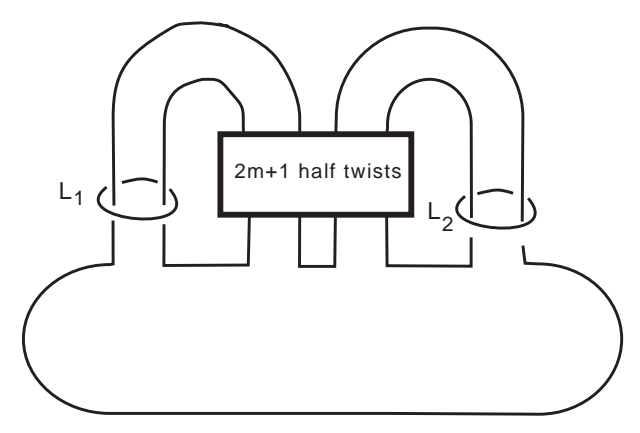

Figure 1: The knot $K_{2 m+1}$

The link $L_{1} \cup L_{2}$ will be used later as follows. A neighborhood of $L_{1}$ will be removed from $S^{3}$ and replaced with the complement of a knot $J$ as described earlier. Similarly, a neighborhood of $L_{2}$ will be removed and replaced with the 
complement of $-J$. This new knot will be denoted $K_{J, 2 m+1}$. As mentioned before, a full explication of this construction is contained in [GL1].

We will need a detailed understanding of the homology of the 3-fold cyclic branched cover of $K_{2 m+1}$. Here we give the general result. The proof appears in detail elsewhere (eg. [GL1]) but because it is simple we give a fairly complete outline.

Let $M_{q}\left(K_{2 m+1}\right)$ denote the $q$-fold cyclic branched cover of $K_{2 m+1}$ and let $\tilde{L}_{i}$ denote a fixed lift of $L_{i}$ to $M_{q}\left(K_{2 m+1}\right), i=1,2$. We will use $\tilde{L}_{i}$ to denote the homology classes represented by the $\tilde{L}_{i}$ also.

Proposition 4.1 $H_{1}\left(M_{q}\left(K_{2 m+1}\right)\right)=\mathbf{Z}_{a} \oplus \mathbf{Z}_{a}$ with $a=\left|(m+1)^{q}-m^{q}\right|$. The homology is generated by the classes represented by $\tilde{L}_{1}$ and $\tilde{L}_{2}$. On homology the deck transformation $T$ acts by $T\left(\tilde{L}_{1}\right)=m^{*}(m+1) \tilde{L}_{1}$ and $T\left(\tilde{L}_{2}\right)=(m+$ $1)^{*} m \tilde{L}_{2}$, where the superscript $\left(^{*}\right)$ denotes the multiplicative inverse modulo $a$.

Proof A formula of Seifert (see [Ro, Section $8 \mathrm{D}]$ ) gives the matrix $\Gamma^{q}-(\Gamma-I)^{q}$ as a presentation matrix for the homology, where $\Gamma=V_{m}\left(V_{m}^{t}-V_{m}\right)$. That $H_{1}\left(M_{q}\left(K_{2 m+1}\right)\right)=\mathbf{Z}_{a} \oplus \mathbf{Z}_{a}$ where $a=\left|(m+1)^{q}-m^{q}\right|$ follows readily.

A Mayer-Vietoris argument used to compute the homology of the cover (again see [Ro, Section 8D]) shows that the $\tilde{L}_{i}$ and their translates generate the homology of the cover and also gives the relations $m T\left(\tilde{L}_{1}\right)=(m+1)\left(\tilde{L}_{1}\right)$ and $(m+1) T\left(\tilde{L}_{2}\right)=m\left(\tilde{L_{2}}\right)$. Hence, $T\left(\tilde{L}_{1}\right)=m^{*}(m+1)\left(\tilde{L}_{1}\right)$ and $T\left(\tilde{L}_{2}\right)=$ $(m+1)^{*} m\left(\tilde{L_{2}}\right)$, as desired. (Notice that $m$ and $m+1$ are invertible modulo $a$ since $m$ (respectively $m+1$ ) and $\left|(m+1)^{q}-m^{q}\right|$ have greatest common divisor 1.)

\section{A positive amphicheiral knot of infinite order in concordance}

Consider the knot $\bar{K}_{2 m+1}=K_{2 m+1} \# K_{2 m+1}^{*}$ illustrated in Figure 2. The knot is easily seen to be strongly positive amphicheiral: reflection through the plane of the page followed by a 180 degree rotation about the axis perpendicular to the plane of the page gives the desired involution.

To form the knot $\bar{K}_{J, 2 m+1}$ we replace neighborhoods of $L_{1}$ and $L_{2}^{\prime}$ with the complement of $J$ and neighborhoods of $L_{2}$ and $L_{1}^{\prime}$ with the complement of 


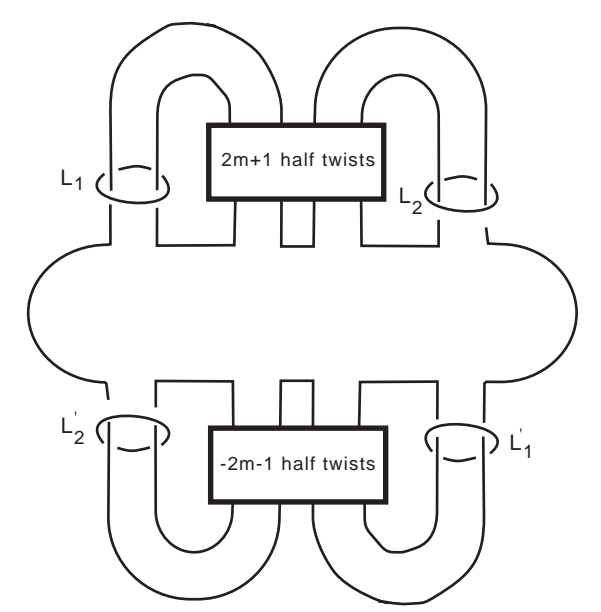

Figure 2: The strongly positive amphicheiral knot, $\bar{K}_{2 m+1}$

$-J$. In this way, $\bar{K}_{J, 2 m+1}=K_{J, 2 m+1} \# K_{J, 2 m+1}^{*}$. (To clarify that the picture is correct, observe that mirror reflection through the plane of the page, followed by a 180 degree rotation about a point in the center of the figure, carries the knot complement that replaces $L_{1}$ to the mirror image of the knot complement that replaces $L_{1}^{\prime}$. Hence, for this knot to be strongly positive amphicheiral the knot complement that replaces $L_{1}$ should be the mirror image of the knot that replaces $L_{1}^{\prime}$.)

Let $p$ be an odd prime dividing $a=(m+1)^{3}-m^{3}$ and suppose that $p$ has exponent 1 in $(m+1)^{3}-m^{3}$. That there is an infinite set of such primes occurring for some $m$ is proved in the appendix. In fact, any prime congruent to 1 modulo 3 suffices.

Proposition 5.1 Suppose that $p$ divides $(m+1)^{3}-m^{3}$ with exponent 1 . The $p$-torsion in the homology of the 3 -fold branched cover of $\bar{K}_{2 m+1}$ is $\left(\mathbf{Z}_{p}\right)^{4}$ generated by the lifts $\left\{\tilde{L}_{1}, \tilde{L}_{2}, \tilde{L}_{1}^{\prime}, \tilde{L}_{2}^{\prime}\right\}$. The deck transformation $T$ has eigenvalues $\lambda_{+}=m^{*}(m+1)$ with eigenvectors $\left\{\tilde{L}_{1}, \tilde{L}_{2}^{\prime}\right\}$ and eigenvalue $\lambda_{-}=(m+1)^{*} m$ with eigenvectors $\left\{\tilde{L}_{2}, \tilde{L}_{1}^{\prime}\right\}$.

Proof This all follows readily from Proposition 4.1.

Before proving the next theorem we need to make a simple number theoretic observation. 
Lemma 5.2 If $p$ is a prime divisor of $\left|(m+1)^{3}-m^{3}\right|$ then $m^{*}(m+1) \neq$ $(m+1)^{*} m \bmod p$.

Proof If $m^{*}(m+1)=(m+1)^{*} m \bmod p$ then $m^{2}=(m+1)^{2} \bmod p$. It cannot be that $m=m+1 \bmod p$, so we would have $m=-m-1 \bmod p$. In other words, $m=-1 / 2 \bmod p$. (Since $p$ is odd, 2 is invertible $\bmod p$.) Substituting this into $(m+1)^{3}-m^{3}$ yields $1 / 4 \bmod p$. But then $(m+1)^{3}-m^{3}$ is clearly not divisible by $p$.

Theorem 5.3 Suppose that some odd prime $p$ divides $(m+1)^{3}-m^{3}$ with exponent 1. For an appropriate choice of $J$ the knot $\bar{K}_{J, 2 m+1}$ has infinite order in the concordance group.

Proof The necessary properties of $J$ will be developed in the course of the proof.

Consider the connected sum of $n$ copies of $\bar{K}_{J, 2 m+1}$. We want to apply the properties of Casson-Gordon invariants as described in Section 3, working with the 3 -fold cover.

Since the $p$-torsion in $H_{1}\left(M_{3}, \mathbf{Z}\right)$ is $\left(\mathbf{Z}_{p}\right)^{4 n}$, the order of $H$ is $p^{2 n}$. Notice that $\left(T-\lambda_{+}\right)\left(T-\lambda_{-}\right)$annihilates the homology of the cover, so $H$ splits into a summand annihilated by $T-\lambda_{+}$and a summand annihilated by $T-\lambda_{-}$. Here we use that the eigenvalues are distinct, which follows from the previous lemma. Call these $H_{+}$and $H_{-}$. One of these has dimension at least $n$; we will assume that it is $H_{+}$, the other case is identical.

Any $v$ in $H_{+}$is a linear combination of the $\left\{\tilde{L}_{1}, \tilde{L}_{2}^{\prime}\right\}$ associated to each of the $n$ summands. We want to see that some such $v$ involves at least $n$ of these basis elements. This is an exercise in elementary linear algebra as follows. Write out a set of basis elements for $H_{+}$in terms of the full set of $\left\{\tilde{L}_{1}, \tilde{L}_{2}^{\prime}\right\}$. Writing these as the rows of a matrix (with at least $n$ rows and exactly $2 n$ columns and applying Gauss-Jordan elimination, combining generators of $\mathrm{H}_{+}$ and reordering the $\left\{\tilde{L}_{1}, \tilde{L}_{2}^{\prime}\right\}$ yields a generating set with matrix representation

$$
\left(\begin{array}{cccc}
1 & 0 & 0 & \ldots \\
0 & 1 & 0 & \ldots \\
0 & 0 & 1 & \ldots \\
\ldots & & &
\end{array}\right) .
$$

The sum of these basis elements corresponding to the rows gives the desired element $v$. 
Linking with $v$ defines a character $\chi$ on the homology of the 3 -fold cover of $n \bar{K}_{2 m+1}$. Since $v$ is in the metabolizer, $\chi$ vanishes on the metabolizer. Considering just a single building block, $K_{2 m+1}$, we have that the linking form satisfies $\operatorname{lk}\left(\tilde{L}_{i}, \tilde{L}_{i}\right)=0, i=1$ and 2 . This implies the $L_{1}$ and $L_{2}$ link nontrivially since the linking form is nonsingular.

From the preceding discussion we see that $\chi$ evaluates nontrivially on at least $n$ of the $\left\{\tilde{L}_{2}, \tilde{L}_{1}^{\prime}\right\}$ and trivially on all of the $\left\{\tilde{L}_{1}, \tilde{L}_{2}^{\prime}\right\}$.

From Proposition 3.2 we have that

$$
\sigma\left(n \bar{K}_{J, 2 m+1}, \chi\right)=\sum_{i} \sum_{j=1}^{3} \sigma_{\alpha_{i, j} / p}(-J) \quad+\sigma\left(n \bar{K}_{2 m+1}, \chi\right) .
$$

In the double summation the index $i$ runs over a set with at least $n$ terms. The $\alpha_{i, j}$ are all nonzero modulo $p$.

The additivity of Casson-Gordon invariants [G2] applied to the second summation yields

$$
\sigma\left(n \bar{K}_{J, 2 m+1}, \chi\right)=\sum_{i} \sum_{j=1}^{3} \sigma_{\alpha_{i, j} / p}(-J)+\sum_{i=1}^{n} \sigma\left(\bar{K}_{2 m+1}, \chi_{i}\right) .
$$

Here the characters $\chi_{i}$ are unknown, but notice that the values of $\sigma\left(\bar{K}_{2 m+1}, \chi_{i}\right)$ are taken from some finite set of rational numbers. Suppose that the maximum value of the absolute values of this finite set of numbers is $C$. Then as long as all of the classical signatures $\sigma_{\alpha / p}(J)$ are greater than $C / 3$, we have that the above sum cannot equal zero. Finding a knot $J$ with this property is simple and the proof is complete.

Refinements A more detailed analysis of $\sigma\left(\bar{K}_{2 m+1}, \chi_{i}\right)$ can be made to show that in fact these terms are all 0 . The idea is that the knots $K_{2 m+1}$ are doubly slice, so that the Casson-Gordon invariants must vanish for both eigenspaces. Since we do not need this to provide examples for the main theorem, we do not include the details here.

It is worth noting one particular case that this refinement addresses, that of Flapan's original example of a nonslice strongly positive amphicheiral knot in [F]. In that case $m=1$ and $J$ is the trefoil. The 3-fold branched cover has homology $\mathbf{Z}_{7} \oplus \mathbf{Z}_{7}$ and we let $p=7$. The associated Casson-Gordon invariants are then classical 7 -signatures of the trefoil. Although not all 7 -signatures are positive, all are nonnegative and the sum will necessarily involve some nonzero terms and is hence nontrivial. Hence Flapan's knot is of infinite order in the concordance group. 


\section{Completion of Theorem 1.1}

\section{An Infinite Family of Linear Independent Examples}

To prove Theorem 1.1 we need to find an infinite family of knots such as those constructed in the previous section, all of which are independent in concordance. The argument is fairly simple, similar to the one by Jiang $[\mathrm{J}]$ giving that the concordance group of algebraically slice knots contains an infinitely generated free subgroup.

Using the results of the appendix, one can find an infinite sequence of integers, $\left\{m_{i}\right\}$, with the property that each $\left(m_{i}+1\right)^{3}-m_{i}^{3}$ is divisible by a prime $p_{i}$ with exponent 1 and no $p_{j}$ divides $\left(m_{i}+1\right)^{3}-m_{i}^{3}$ if $i \neq j$.

Next construct the $\bar{K}_{J_{i}, 2 m_{i}+1}$ as in the previous section. We observe that the $\bar{K}_{J_{i}, 2 m_{i}+1}$ form the desired set, as follows. Suppose that some linear combination of these was slice. Let $\bar{K}_{J_{n}, 2 m_{n}+1}$ be one of the knots in that linear combination. Apply the Casson-Gordon result, Proposition 3.2, using characters on the 3 -fold cover to $\mathbf{Z}_{p_{n}}$. Using additivity, the value of the Casson-Gordon invariants is reduced to that on the 3 -fold cover of the multiple of $\bar{K}_{m_{n}}$. (The character vanishes on the covers of the other summands by the choice of the $p_{i}$ so Proposition 3.3 applies.) The calculations of the previous section show that some such Casson-Gordon invariants do not vanish.

\section{Primeness}

The proof of Theorem 1.1 is now completed by showing that each of the $\bar{K}_{J_{i}, 2 m_{i}+1}$ is concordant to a prime knot. In [F] Flapan showed that one particular example of a $\bar{K}_{J, 2 m+1}$ is concordant to a prime knot that is strongly positive amphicheiral. Her proof applies in the present setting. This completes the proof.

\section{A Appendix: Prime divisors of $(m+1)^{3}-m^{3}$}

Theorem A.1 The set of primes that divide $F(m)=(m+1)^{3}-m^{3}=3 m^{2}+$ $3 m+1$ for some $m$ is infinite.

Proof The proof is reminiscent of Euclid's proof of the infinitude of primes. Suppose that the set of such primes is finite, $\left\{p_{i}\right\}_{i=1, \ldots, n}$. Let $N=\prod_{i=1, \ldots, n} p_{i}$. Consider $F(N)$. Clearly none of the $p_{i}$ divide $F(N)=3 N^{2}+3 N+1$, so there is a prime factor of $F(N)$ that is not among the $p_{i}$, contradicting the definition of $\left\{p_{i}\right\}$. 
Theorem A.2 The set of primes $p$ for which $p$ is a prime divisor of $F(m)$ with exponent one for some $m$ is infinite.

Proof We prove that, in fact, if a prime divides $F(m)$ for some $m$, then it divides $F(m+p)$ with exponent 1. Suppose that $p$ has exponent greater than 1 in $F(m)$. Use Taylor's theorem to find $F(m+p)=F(m)+p F^{\prime}(m)+p^{2} F^{\prime \prime}(m) / 2$. ( $F$ is quadratic.) The first and last terms are divisible by $p^{2}$, so $p$ has exponent 1 in $F(m+p)$ unless $p$ divides $F^{\prime}(m)$. But, if $F(m)$ and $F^{\prime}(m)$ have a common root then $F(m)$ would have multiple root. But this can occur if and only if the discriminant of this quadratic is 0 . In this case the discriminant is -3 , so a multiple root does not occur unless $p=3$. (As an alternative, to see that $F(m)$ and $F^{\prime}(m)$ do not have a common root just note that a simple calculation shows that $4 F(m)-(2 m+1) F^{\prime}(m)=1$ for all $m$, so that $p$ cannot divide both $F(m)$ and $F^{\prime}(m)$.

Although we don't need a particular description of the primes that occur, such a calculation is possible.

Theorem A.3 A prime $p$ occurs as a divisor of $F(m)$ with exponent 1 for some $m$ if and only if $p$ is congruent to $1 \bmod 3$.

Proof By the previous theorem we need not concern ourselves with the exponent of $p$. Hence, we want the conditions for $3 m^{2}+3 m+1$ to have a root modulo $p$. But, using the quadratic formula we quickly see that this will occur if and only if there is square root of -3 in $\mathbf{Z}_{p}$. Using the language of quadratic symbols $[\mathrm{La}]$, we are asking for which $p$ we have $\left(\frac{-3}{p}\right)=1$.

From the properties of the quadratic symbol we have

$$
\left(\frac{-3}{p}\right)=\left(\frac{-1}{p}\right)\left(\frac{3}{p}\right)=(-1)^{\frac{p-1}{2}}\left(\frac{3}{p}\right) .
$$

Applying quadratic reciprocity (again, see [La]) to the second term gives

$$
(-1)^{\frac{p-1}{2}}\left(\frac{3}{p}\right)=(-1)^{\frac{p-1}{2}}\left(\frac{p}{3}\right)(-1)^{\left(\frac{p-1}{2}\right)\left(\frac{3-1}{2}\right)}=\left(\frac{p}{3}\right) .
$$

This last term is 1 if and only if $p$ is a square modulo 3 , which occurs if and only if $p$ is congruent to 1 modulo 3 . 


\section{References}

[CG1] A Casson, C Gordon, Cobordism of classical knots, in $A$ la recherche de la Topologie perdue, ed. by Guillou and Marin, Progress in Mathematics, Volume 62, 1986, pp.181-199. (Originally published as Orsay Preprint, 1975.)

[CM] D Coray, M Daniel, Knot cobordism and amphicheirality, Comment. Math. Helv. 58(4) (1983) 601-616.

[F] E Flapan, A prime strongly positive amphicheiral knot which is not slice, Math. Proc. Cambridge Philos. Soc. 100(3) (1986) 533-537.

[G2] P Gilmer, Slice knots in $S^{3}$, Quart. J. Math. Oxford Ser. (2) 34(135) (1983) 305-322.

[GL1] P Gilmer, C Livingston, The Casson-Gordon invariant and link concordance, Topology 31(3) (1992) 475-492.

[G] C McA Gordon, Problems in Knot Theory, in Knot Theory, ed. J. Hausmann, Springer Lecture Notes no. 6851977.

[HK] R Hartley, A Kawauchi, Polynomials of amphicheiral knots, Math. Ann. 243(1) (1979) 63-70.

[J] B Jiang, A simple proof that the concordance group of algebraically slice knots is infinitely generated, Proc. Amer. Math. Soc. 83 (1981) 189-192.

[La] S Lang, Algebraic Number Theory, Addison-Wesley Publishing Co., Reading, Mass., 1968.

[Lt] $\quad$ R Litherland, Cobordism of satellite knots, in Four-Manifold Theory, Contemporary Mathematics, eds. C. Gordon and R. Kirby, American Mathematical Society, Providence RI 1984, 327-362.

[L1] C Livingston, Knots which are not concordant to their reverses, Oxford Q. J. of Math. 34 (1983), 323-328.

[L2] C Livingston, Examples in concordance, preprint at http://front.math.ucdavis.edu/math.GT/0101035.

[Lo] D D Long, Strongly plus-amphicheiral knots are algebraically slice, Math. Proc. Cambridge Philos. Soc. 95(2) (1984) 309-312.

[Ro] D Rolfsen, Publish or Perish, Berkeley CA (1976).

Department of Mathematics, Indiana University

Bloomington, IN 47405, USA

Email: livingst@indiana.edu

Received: 17 December 2000 